This item was submitted to Loughborough's Research Repository by the author.

Items in Figshare are protected by copyright, with all rights reserved, unless otherwise indicated.

\title{
A new freeware tool for image processing and its application to high speed LA-ICP-MS imaging
}

\section{PLEASE CITE THE PUBLISHED VERSION}

https://doi.org/10.1039/c9ja00082h

\section{PUBLISHER}

(c) Royal Society of Chemistry

\section{VERSION}

AM (Accepted Manuscript)

\section{PUBLISHER STATEMENT}

This paper was accepted for publication in the journal Journal of Analytical Atomic Spectrometry and the definitive published version is available at https://doi.org/10.1039/c9ja00082h.

LICENCE

CC BY-NC-ND 4.0

\section{REPOSITORY RECORD}

Managh, Amy, and Peter Reid. 2019. "A New Freeware Tool for Image Processing and Its Application to High Speed LA-ICP-MS Imaging". figshare. https://hdl.handle.net/2134/37621. 
Received 00th January 20xx, Accepted 00th January 20xx

DOI: $10.1039 / \times 0 \times x 00000 x$

www.rsc.org/jaas

\section{A new freeware tool for image processing and its application to high speed LA-ICP-MS imaging}

\author{
Amy J. Managh*a and Peter Reid ${ }^{\mathrm{b}}$ \\ A new standalone, non-commercial software application has been developed to enable rapid generation of images from \\ laser ablation - inductively coupled plasma - mass spectrometry data. The software locates peaks within raw data files \\ and uses the peak locations to segment the data at appropriate intervals, enabling conversion of the data into a matrix of \\ colour coded pixels. The app was developed in order to process baseline separated peaks produced by the latest \\ generation of low-dispersion laser ablation systems, but it is equally applicable to rastered imaging using alternative laser \\ ablation platforms. The peak recognition feature also has applicability to single cell and single particle ICP-MS \\ applications. The app was demonstrated for the imaging of zinc in a stained section of rat retina tissue at lateral \\ resolutions down to $1 \mu \mathrm{m}$. Less than two minutes was required for conversion of the raw data into a 60,000 pixel image.
}

\section{Introduction}

Laser ablation - inductively coupled plasma - mass spectrometry (LA-ICP-MS) is a highly sensitive technique for elemental imaging that is well established in the fields of biological and earth sciences. Until recently, the most common approach to acquire LA-ICP-MS images has been 'rastering'. This involves ablating lines of overlapping craters on the sample surface, usually with a laser repetition frequency that far exceeds the temporal dispersion of ablated material through the system. ${ }^{1}$ Thus, rastering results in mixing of the material produced from successive laser shots and the resulting pixels in the image are blurred/distorted from the spatial coordinates that they represent. With recent growth in the analysis of discrete small-volume samples, notably of single cells, ${ }^{2,3}$ the need to preserve spatial resolution is becoming an increasingly important factor in imaging applications. One solution is to recover the resolution lost through rastering at the data processing stage using image deconvolution software. ${ }^{4}$ However, the past decade has also seen significant advancements in the design of laser ablation chambers and their interface to the ICP-MS, 1,5 which presents opportunities to re-evaluate the way in which laser ablation data is acquired. These developments have led to a dramatic improvement in the transport characteristics of the ablated aerosol. In some cases the signal durations produced from a single laser shot have reduced from several hundred

\footnotetext{
a. Department of Chemistry, Loughborough University, LE11 3TU, UK.

b. Reid-IT Limited, Loughborough, Leicestershire, LE11 3SP, UK.

† Footnotes relating to the title and/or authors should appear here.

Electronic Supplementary Information (ESI) available: [details of any supplementary information available should be included here]. See
} DOI: $10.1039 / x 0 x \times 00000 x$ milliseconds down to less than 10 milliseconds. ${ }^{6,7}$ As a result, sampling can occur at a much higher rate than previously possible and consequently the use of 'pixel-by-pixel' sampling, rather than rastering is becoming feasible. ${ }^{8}$ In this approach the signal from each laser shot is allowed to wash out of the system before the next adjacent location is sampled, producing baseline resolved signals. The data under each peak is integrated and converted to an individual colour coded pixel that directly represents the location that was sampled; hence resolution is preserved at the data acquisition stage.

A range of both in-house and commercial software programs exist for the processing of LA-ICP-MS data, including GLITTER, ${ }^{9}$ iQuant $2,{ }^{10}$ LADR, ${ }^{11}$ HDIP, ${ }^{12}$ and LA-iMageS. ${ }^{13}$ A range of scripts that run inside other commercial platforms are also available, including lolite which runs in IGOR Pro, ${ }^{14}$ SILLS which runs in MATLAB ${ }^{15}$ and TERMITE which runs in R. ${ }^{16}$ The majority of these were developed to work with rastered data, thus they are limited in their ability to handle data generated using a 'pixel-by-pixel' imaging approach. Some of the newer platforms address a wider variety of image acquisition methods, but so far these are proprietary systems and have not yet been adopted for mainstream use. This article therefore discusses the development of a freeware software package, LA-ICP-MS Image Tool, which is tailored to the generation of images from pixel-by pixel imaging data. The software also has the capability to perform identification of peaks in single-shot or single particle ICP-MS experiments, which is useful in the alignment of line scans. The capabilities of the software are illustrated using an example of retinal imaging. The retina contains a range of small scale structural features, the elemental composition of which is pertinent in the study of age-related macular degeneration. ${ }^{17}$ RodríguezMenéndez et al. recently reported LA-ICP-MS imaging of zinc in the human retina with a $25 \mu \mathrm{m}$ spot size and a rastered 
imaging approach. ${ }^{18}$ In the present article, a pixel-by-pixel approach and the Image Tool are instead applied to provide higher resolutions, down to $1 \mu \mathrm{m}$, in much smaller sized rat retinal samples. This represents the first application of a nominal $1 \mu \mathrm{m}$ spot size to LA-ICP-MS imaging of retinal tissue.

\section{Features of the Image Tool}

The Image Tool was created using the LiveCode development environment. ${ }^{19}$ It required approximately 9500 lines of code and went through 68 versions with an estimated development effort of 10 man-weeks coding time before its current release. The final version of the executable program can be freely downloaded (https://www.reidit.co.uk/downloads/down la icp ms it.htm). The software has three main windows. The main functions of these is to 1 ) import data files and locate peaks within them, 2) segment the data at desired intervals to generate a matrix of pixels and 3 ) view and save the resulting images.

The software has been pre-programmed to accept txt and csv based source files from a variety of ICP-MS instruments. Also, the importer can be manually modified within the user interface to accept additional file formats. Data files are imported by selection of a folder containing each line of the image within a separate data file. Following import, the software parses each line of the image individually and it initially stores the timestamped data in one dimensional arrays. Since the majority of analyses are performed using a trigger cable between the LA and ICP-MS units, the data of interest will usually start to appear at the same relative point within each data file. This point can be directly specified within the user interface to aid segmenting of the file. Alternatively, for analyses performed without a trigger cable, the software includes a peak recognition feature, which identifies peaks based on user defined characterisation of a typical peak height and width. Information about the peaks identified within each experiment can be extracted into an Excel file. Thus, the software has applicability to single cell and single particle ICP-MS experiments, in addition to LA-ICP-MS imaging.

The second screen displays a list of the data files processed and, where peak recognition has been used, displays the time point of the first peak located within each file. This information can be exported as a table and, if necessary, the user can select to either re-run peak recognition on certain files using different settings or to manually override the start time for selected files. Once the start time has been determined, the data can then be segmented into a twodimensional array of pixels according to user defined criteria. The methodology used to segment the data is presented schematically in Figure 1. Firstly, using the location of the first peak within each file, the integration windows are positioned so that the peaks lie within them. Secondly, the counts within each window are summed to give a single value per sampled location. These values are then converted into a 2D matrix of data containing the values for the pixels in the image. Finally,
Step 1: Peak recognition and characterisation of data

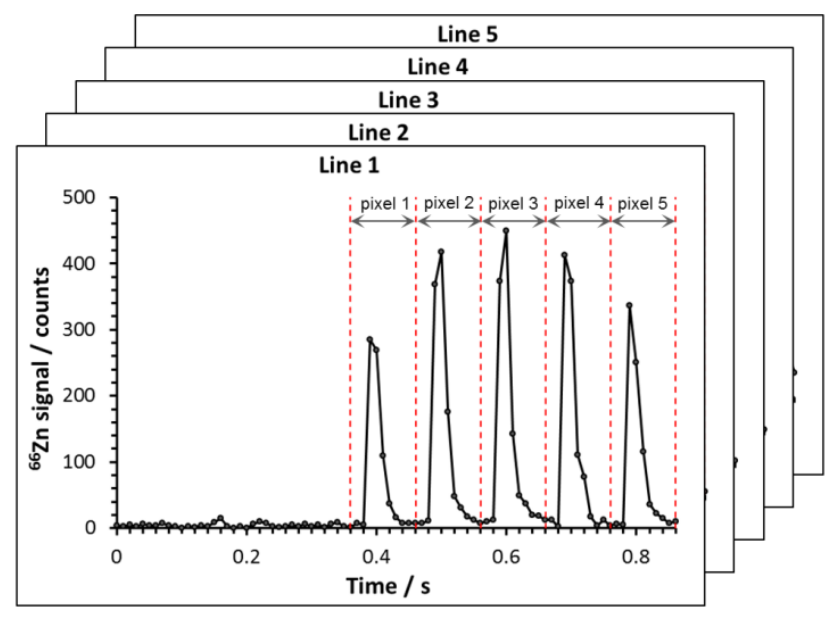

Step 2: Generation of data matrix

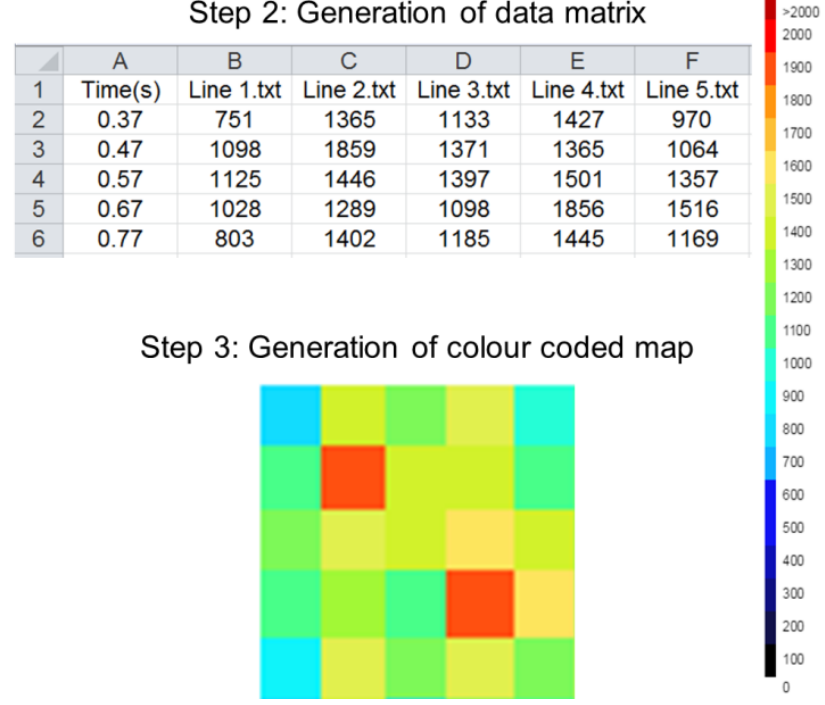

Fig. 1 Schematic overview of the LA-ICP-MS Image Tool showing an extract of data obtained following ablation of a gelatine standard at $10 \mathrm{~Hz}$. The first peak is found within each file and this is used to position the windows (dotted red lines). The counts within each window are then summed to create a matrix of data values for each laser shot (step 2), which is converted into a colour coded image (step 3).

the data matrix is converted into colour coded pixels. The default colour scheme is a rainbow format, but the choice of colour palette is customisable and is based on hexadecimal coding. The viewer window also provides the opportunity to perform basic background subtraction based on a linear interpolation up to three background regions and to calculate basic ratios between two measured elements or isotope channels. The final image may be either exported directly as a png image or exported as a .txt or .csv document for further processing or input another graphics package. 


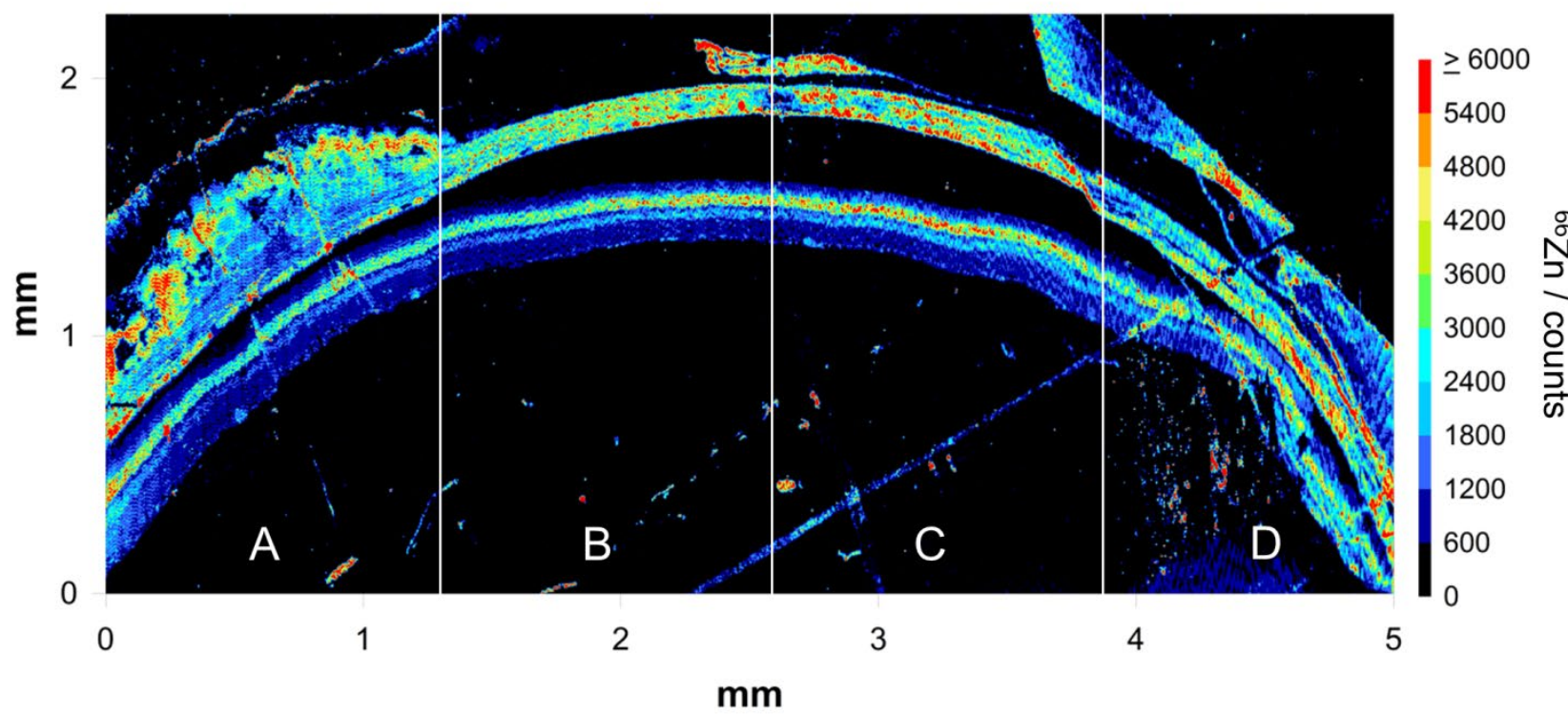

Fig. 2 Image of 4 consecutive sections of retina tissue, sampled using a $5 \mu \mathrm{m}$ spot, with different repetition frequencies and scan speeds. A) $10 \mathrm{~Hz}$ and $50 \mu \mathrm{m} / \mathrm{s}$, B) $20 \mathrm{~Hz}$ and $100 \mu \mathrm{m} / \mathrm{s}, \mathrm{C}) 50 \mathrm{~Hz}$ and $250 \mu \mathrm{m} / \mathrm{s}, \mathrm{D}) 100 \mathrm{~Hz}$ and $500 \mu \mathrm{m} / \mathrm{s}$.

\section{Demonstration for Retinal Imaging}

Zinc is an essential trace element in the eye and its distribution and deposition within the structures of the eye has been linked with the progression and treatment of several visual disorders. $^{20}$ The retina is a complex structure, composed of several thin layers, that lines the back of the inner eye. In rat samples these layers are in the order of tens of micrometres in thickness, so the ability to maintain high spatial resolution when imaging these features is essential. Consequently imaging retina samples provides an effective illustration of the importance of lateral resolution in LA-ICP-MS. Post mortem retina samples taken from 18 month old female Long Evans rats were obtained from the Institute of Ophthalmology, University College London. Samples were sectioned to $10 \mu \mathrm{m}$ thickness and were stained with haematoxylin prior to analysis to aid visualisation of the small structural features within them.

Analysis of the samples was performed using an NWR image laser ablation system (Elemental Scientific Lasers, Bozeman, USA) coupled to a sector field ICP-MS (Element XR, Thermo Fisher Scientific, Bremen, Germany). The laser ablation system was fitted with a Dual Concentric Injector (DCI) to produce fast transient signals. A helium flow rate of $1.3 \mathrm{l} / \mathrm{min}$ was used through the ablation chamber, with an argon make-up flow rate of $0.5 \mathrm{l} / \mathrm{min}$ introduced through the $\mathrm{DCl}$. An external data acquisition board (P7882, FAST ComTec $\mathrm{GmbH}$, Oberhaching, Germany) was connected to the ion detection board of the Element XR in order to collect blind time free data at $10 \mathrm{~ms}$ intervals. A description of the acquisition board and the connections required to interface it to the ICP-MS has been described in detail previously. ${ }^{21}$ The resulting data was processed into images using the LA-ICP-MS Image Tool.

In order to investigate the compromise between resolution and acquisition speed, a range of different scan speeds and repetition rates were used to image four consecutive sections of a retina sample. In each case the conditions were chosen to ensure that a new sample location was ablated per laser shot (see Table 1). The fluence was optimised to ensure complete ablation through the tissue section, whilst minimising ablation of the glass substrate. The typical washout of the signal was $30-40 \mathrm{~ms}$ at full width $10 \%$ maximum, so baseline resolved signals were observed between peaks at $10 \mathrm{~Hz}$. Under this condition there were 10 data points acquired per laser shot and these were combined into a single pixel using the Image Tool. In contrast, at $100 \mathrm{~Hz}$ the material from consecutive laser shots fully overlapped to create a smoother signal profile, similar to that of a typical raster scan. At this condition the time resolution of the data output was equivalent to the laser firing rate, hence each data point was treated as a pixel in the final image. Figure 2 shows a side-by-side comparison of images generated from this experiment. Five distinct layers can be observed in the retina under all resolutions studied.

Table 1 Experimental conditions used for the initial low resolution analysis of the retina sample.

\begin{tabular}{lcccc}
\hline LA Parameter & $\mathrm{A}$ & $\mathrm{B}$ & $\mathrm{C}$ & $\mathrm{D}$ \\
\hline Fluence $/ \mathrm{J} \mathrm{cm}^{-2}$ & 15 & 15 & 15 & 15 \\
Spot size $/ \mu \mathrm{m}$ & 5 & 5 & 5 & 5 \\
Repetition frequency / Hz & 10 & 20 & 50 & 100 \\
Scan speed / $\mathrm{\mu} \mathrm{s}^{-1}$ & 50 & 100 & 250 & 500 \\
\hline Image Tool Parameter & $\mathrm{A}$ & $\mathrm{B}$ & $\mathrm{C}$ & $\mathrm{D}$ \\
\hline Data intervals / s & 0.01 & 0.01 & 0.01 & 0.01 \\
Image Tool segment / s & 0.1 & 0.05 & 0.02 & 0.01 \\
Data points per pixel & 10 & 5 & 2 & 1 \\
\hline
\end{tabular}


There is no perceptible loss in resolution between the 10 and $20 \mathrm{~Hz}$ conditions. However, inspection of the higher repetition rates reveals that, as expected, there is a minor loss of definition between the outer and inner nuclear layers at the higher scan speed. The average width of the outer nuclear layer was subjectively determined in each segment of the image using the distance measurement feature in DPlot (HydeSoft Computing, USA). The average widths were 53.7, $54.3,56.7$ and $58.9 \mu \mathrm{m}$ for the 10, 20, 50 and $100 \mathrm{~Hz}$ sections respectively. This equates to a $5.2 \mu \mathrm{m}$ difference between the 10 and $100 \mathrm{~Hz}$ settings, approximately equivalent to the width of the laser spot size used here.

A subsequent higher resolution image was obtained using a 1 $\mu \mathrm{m}$ spot, $10 \mu \mathrm{m} / \mathrm{s}$ scan speed, $10 \mathrm{~Hz}$ repetition rate and laser fluence of $15 \mathrm{~J} / \mathrm{cm}^{2}$. The resulting image is shown in Figure 3. In this image we can see that the outer and inner nuclear layers are not a continuous band, but are comprised of a series of cells. Individual cells of around $5 \mu \mathrm{m}$ diameter can be clearly distinguished within the outer nuclear layer and these match closely with the microscopic image. Closer inspection of the microscopic image also reveals the inhomogeneity of the stain within the inner plexiform and photoreceptor layers and this pattern is also observed in the LA-ICP-MS image. The section imaged for this study was stained with a substance known to contain traces of zinc, so the image in the present study cannot be used to make conclusions about the distribution of zinc in age related macular degeneration. However, this example provides indicative evidence of the level of spatial
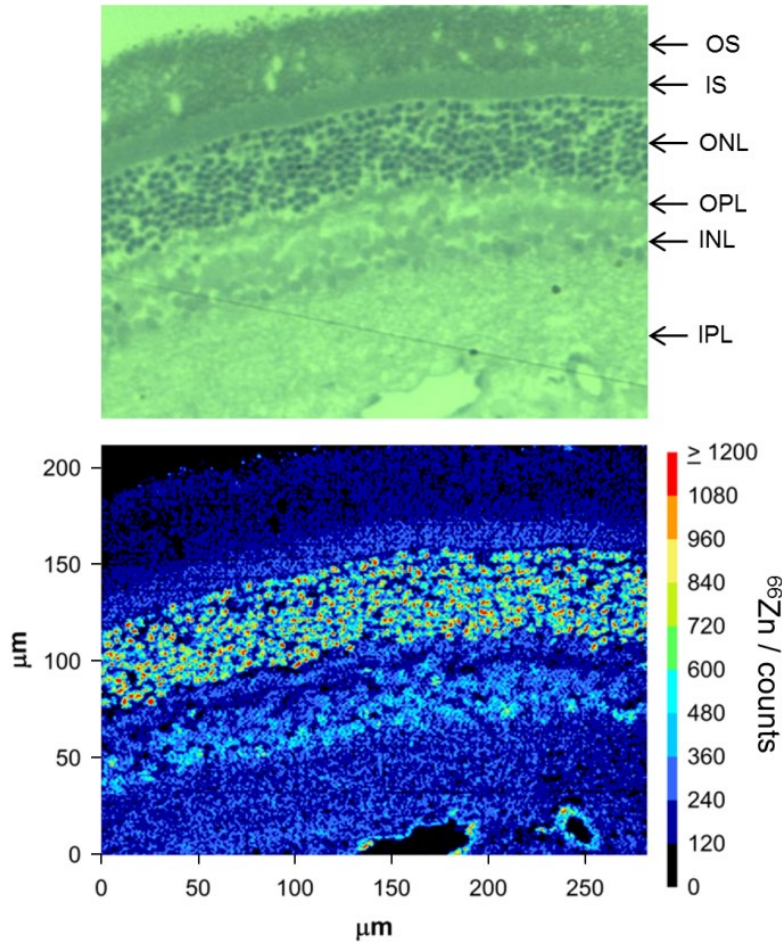

Fig. 3 Top: Microscopic image of a section of retina tissue showing the outer segment (OS), inner segment (IS), outer nuclear layer (ONL), outer plexiform layer (OPL), inner nuclear layer (INL) and inner plexiform layer (IPL). Bottom: High resolution LA-ICP-MS image of $\mathrm{Zn}$ distribution in the above section obtained using a $1 \mu \mathrm{m}$ spot, $10 \mathrm{~Hz}$ repetition rate and $10 \mu \mathrm{m} / \mathrm{s}$ scan speed. resolution that may be possible in such studies in the future.

\section{Conclusions}

This technical note presents new software, the "LA-ICP-MS Image Tool", for converting raw LA-ICP-MS data into images. The software operates as a standalone application and can be downloaded and operated freely without licence. Imaging was demonstrated using data acquired on a biological specimen under a variety of conditions, which provided both continuous signals and baseline separated peaks. The software therefore presents a range of options to users when considering appropriate methods to acquire and process their data. Importantly, one of the major advantages of the latest generation laser ablation platforms, namely their potential for high speed imaging, is not negated by excessive data processing time. The time taken from opening the Image Tool to obtaining a preview of the $\sim 60,000$ pixel image shown in Figure 3 was less than two minutes, indicating that the Image Tool is a viable approach for use with high throughput imaging applications. The software does not currently support complex processing for specialised applications, for example interference or mass bias correction. However, the image matrix may be exported as an Excel compatible file, allowing further processing to be carried out off-line if required.

\section{Conflicts of interest}

There are no conflicts to declare.

\section{Acknowledgements}

The authors gratefully acknowledge Imre Lengyel and colleagues for donation of the retina sample used in this work. The authors would also like to thank Andrea de Bruin, Kelly Spencer and Calum Greenhalgh for their help during acquisition of the sample data sets, Helen Reid (Loughborough University), Grant Craig (Thermo Fisher Scientific) and Matthew Horstwood (British Geological Survey) for their advice during development and testing of the software, and Tharwat Abduljabbar (KAUST) for discussions about retina imaging.

\section{References}

1 S.J.M. Van Malderen, A.J. Managh, B.L. Sharp and F. Vanhaecke, J. Anal. At. Spectrom., 2016, 31, 423-439.

2 A. J. Managh, R. W. Hutchinson, P. Riquelme, C. Broichhausen, A. K. Wege, U. Ritter, N. Ahrens, G. E. Koehl, L. Walter, C. Florian, H. J. Schlitt, H. J. Reid, E. K. Geissler, B. L. Sharp and J. A. Hutchinson, J. Immunol., 2014, 193, 26002608.

3 J. Pisonero, D. Bouzas-Ramos, H. Traub, B. Cappella, C. Álvarez-Llamas, S. Richter, J.C. Mayo, J.M. Costa-Fernandez and N. Jakubowski, J. Anal. At. Spectrom., 2019, DOI: 10.1039/C8JA00096D.

4 S.J.M. van Malderen, J.T. van Elteren and F. Vanhaecke, Anal. Chem., 2015, 87, 6125-6132. 
5 A. Gundlach-Graham and D. Günther, Anal. Bioanal. Chem., 2016, 408, 2687-2695.

6 D. N. Douglas, A. J. Managh, H. J. Reid and B. L. Sharp, Anal. Chem., 2015, 87, 11285-11294.

7 S.J.M. Van Malderen, J.T. van Elteren and F. Vanhaecke, J. Anal. At. Spectrom., 2015, 30, 119-125.

8 M. Burger, A. Gundlach-Graham, S. Allner, G. Schwartz, H.A.O. Wang, L. Gyr, S. Burgener, B. Hattendorf, D. Grolimund and D. Günther, Anal. Chem., 2015, 87, 82598267.

9 W.L. Griffin, W. Powell, N.J. Pearson and S.Y. O'Reilly, in Laser Ablation-ICP-MS in the Earth Sciences. Current Practices and Outstanding Issues, ed. P. Sylvester. Short Course Series, Mineral Association of Canada. 2008, 40, 308311.

10 T. Suzuki, S. Sakata, Y. Makino, H. Obayashi, S. Ohara, K. Hattori and T. Hirata, Mass Spectrom. (Tokyo), 2018, 7, A0065.

11 A. Norris, Laser Ablation Data Reduction Software, https://norris.org.au/ladr/, (accessed March 2019).

12 S.J.M. Van Malderen, presented in part at $14^{\text {th }}$ European Workshop on Laser Ablation, Pau, June, 2018.

\section{Graphical Abstract}

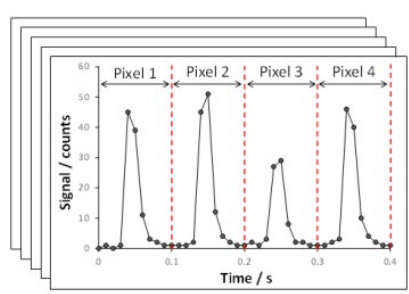

LA-ICP-MS Image Tool

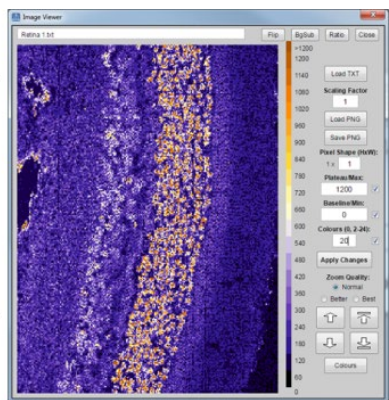

New LA-ICP-MS imaging software has been developed and applied to perform fast, high spatial resolution imaging of zinc in retina tissue.
13 H. López-Fernández, G. de S. Pessôa, M.A.Z. Arruda, J.L. Capelo-Martínez, F. Fdez-Riverola, D. Glenz-Peña and M. Reboiro-Jato, J. Cheminform. 2016, 8, 65-74.

14 C. Paton, J. Hellstrom, B. Paul, J. Woodhead and J. Hergt, J. Anal. At. Spectrom., 2011, 26, 2508-2518.

15 M. Guillong, D.L. Meier, M.M. Allan, C.A. Heinrich and B.W.D. Yardley, in Laser Ablation-ICP-MS in the Earth Sciences. Current Practices and Outstanding Issues ed. P. Sylvester, Short Course Series, Mineral Association of Canada, 2008, 40, 328-333.

16 S.A. Mischel, R. Mertz-Kraus, K.P. Jochum and D. Scholz, Rapid Commun. Mass Spectrom., 2017, 31, 1079-1087.

17 R. Vishwanathan, M. Chung and E.J. Johnson, Investigative Opthalmology and Visual Science, 2013, 54, 3985-3998.

18 S. Rodríguez-Menéndez, B. Fernández, M. García, L. Álvarez, ML. Fernández, A. Sanz-Medel, M. Coca-Prados, R. Pereiro and H. González-Iglesias. Talanta, 2018, 178, 222-230.

19 LiveCode, https://livecode.com, (accessed March 2019).

20 I. Lengyel, J.M. Flynn, T. Peto, D.H. Linkous, K. Cano, A.C. Bird, A. Lanzirotti, C.J. Frederickson and F.J. van Kuijk, Exp. Eye Res., 2007, 84, 772-780.

21 A. J. Managh, D. N. Douglas, K. M. Cowen, H. J. Reid and B. L. Sharp, J. Anal. At. Spectrom., 2016, 31, 1688-1692. 